\title{
HIGH-RESOLUTION H I OBSERVATIONS OF THE GALAXY NGC 404: A DWARF S0 WITH ABUNDANT INTERSTELLAR GAS
}

\author{
M. S. DEL Río \\ Instituto Nacional de Astrofísica, Optica y Electrónica, Tonantzintla, 72000 Puebla, Mexico; and Universidad Popular Autónoma del Estado de Puebla, \\ 72160 Puebla, Mexico; sole@inaoep.mx \\ E. BRINKS \\ Instituto Nacional de Astrofísica, Optica y Electrónica, Tonantzintla, 72000 Puebla, Mexico; ebrinks@inaoep.mx \\ AND \\ J. CEPA ${ }^{1}$ \\ Instituto de Astrofísica de Canarias, E-38200 La Laguna, Tenerife, Spain; jcn@1l.iac.es \\ Received 2003 June 4; accepted 2004 March 16
}

\begin{abstract}
As part of a detailed study of the gas content in a sample of early-type galaxies, we present $21 \mathrm{~cm} \mathrm{H} \mathrm{I} \mathrm{line} \mathrm{maps}$ of the S0 galaxy NGC 404 at a spatial resolution of $15^{\prime \prime} 2^{\prime} \times 14.4(\alpha \times \delta)$ and a velocity resolution of $2.6 \mathrm{~km} \mathrm{~s}^{-1}$. The $\mathrm{H}$ I has been traced out to a radius $R \sim 8 R_{25}$ or 48 disk scale lengths, making it one of the largest $\mathrm{H}$ I extents reported $\left(800^{\prime \prime}\right.$ or $12.6 \mathrm{kpc}$ at the assumed distance of $\left.3.3 \mathrm{Mpc}\right)$. Approximately $75 \%$ of the $\mathrm{H}$ I resides in a doughnut, which is seen close to face-on with inner and outer radii of $\sim R_{25}$ and $\sim 4 R_{25}$, respectively. The optical galaxy fits snugly within the hole of the doughnut. The remaining $25 \%$ of the neutral gas is found in an annulus concentric with the doughnut and with a somewhat larger ellipticity, extending from a radius of $\sim 4 R_{25}$ to $\sim 8 R_{25}$. A total $\mathrm{H}$ I mass of $1.52 \pm 0.04 \times 10^{8} M_{\odot}$ is found, which implies an $M_{\mathrm{HI}} / L_{B}=0.22$ in solar units. We argue that most, if not all, of this gas is of external origin, most likely from the merger of a dwarf irregular galaxy with $M_{B} \sim-15.5$ mag. The velocity field shows a steeply declining observed rotation curve, compatible with Keplerian decline. However, because the galaxy is close to face-on, there is a degeneracy in the determination of the intrinsic rotation curve and inclination. We therefore analyzed two extreme cases, producing tilted-ring model fits forcing either a Keplerian or a flat rotation curve through the observations; both approaches result in equally plausible fits. In both model fits, the position angle of the kinematical major axis of the annulus is distinct from that of the doughnut and ranges from $160^{\circ}$ to $120^{\circ}$ (for the doughnut these values are $100^{\circ}$ to $60^{\circ}$ ). Assuming a distance of $3.3 \mathrm{Mpc}$, a total mass of $3 \times 10^{10} M_{\odot}$ is found on the basis of the Keplerian rotation curve. This implies a global $M_{T} / L_{B}$ ratio of $\sim 44$ in solar units, which is high and likely a reflection of the low blue luminosity of the galaxy ( $\sim 15$ times lower than the average S0 luminosity). Values for a flat rotation curve are a factor of 4 higher.
\end{abstract}

Key words: galaxies: elliptical and lenticular, cD — galaxies: individual (NGC 404) galaxies: kinematics and dynamics - galaxies: structure - radio lines: galaxies

\section{INTRODUCTION}

Traditionally, S0 galaxies were thought to represent a transition between elliptical (E) and spiral (S) galaxies. They share with the latter a central bulge and an exponential disk, and with the former the lack of spiral structure or arms. They are considered inert objects with very little gas, composed almost exclusively of Population II stars, and virtually a total absence of ongoing star formation. This picture has changed considerably over the past $20 \mathrm{yr}$ thanks mainly to tremendous progress in observational techniques, which are now routinely delivering high-sensitivity data over a wide range of wavelengths.

The interstellar medium of early-type galaxies, and especially that of $\mathrm{S} 0 \mathrm{~s}$, has attracted a great deal of interest ever since Faber \& Gallagher (1976) showed that a considerable amount of mass ejected by evolved stars could pile up over the lifetime of the galaxy. Surprisingly, although high-resolution, sensitive $\mathrm{H}$ I observations demonstrated that in general hardly

\footnotetext{
${ }^{1}$ Departamento de Astrofísica, Facultad de Física, Universidad de La Laguna, E-38071 La Laguna, Tenerife, Spain.
}

any gas is detected and that most of it seems to have been accreted (van Gorkom et al. 1986; Wiklind \& Henkel 1990). The scarcity of material detected in $\mathrm{H}, \mathrm{H} \alpha, \mathrm{CO}$, and at FIR wavelengths, and the origin of that material form two puzzles of contemporary astronomy.

Faber \& Gallagher (1976) discuss several ways in which gas could have been expelled or eliminated from early-type systems. They reach the conclusion that gas is particularly hard to remove from these systems via star formation. Although star formation has virtually ceased, there are indications that some star formation is taking place in S0s, from IRAS (Jura 1986; Knapp et al. 1989) and CO observations (Wiklind \& Henkel 1989; Sage 1990), which show that some S0s rival spirals in their CO content, but that the molecular gas is concentrated within 1-2 kpc from the center.

We have started a project, mapping a few early-type galaxies in detail, in order to shed light on the origin of the ISM in these objects. In this paper we present the results obtained for one of the galaxies under study, NGC 404.

NGC 404 is a typical example of an early-type galaxy, which shows more activity than expected from such systems. 
TABLE 1

Optical Parameters of NGC 404

\begin{tabular}{|c|c|}
\hline Parameter & Value \\
\hline Morphological type.. & S0 \\
\hline 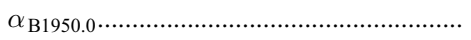 & 010639.0 \\
\hline 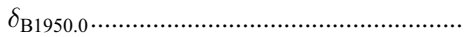 & 352706.0 \\
\hline Isophotal major diameter $D_{25} \ldots \ldots \ldots \ldots \ldots$ & $3.5^{\mathrm{a}}$ \\
\hline Exponential scale length $B \alpha^{-1}$. & $16^{\prime \prime} 56^{\mathrm{b}}$ \\
\hline Exponential scale length $V \alpha^{-1} \ldots \ldots \ldots \ldots \ldots$ & $129^{\prime \prime} 5^{\mathrm{c}}$ \\
\hline Inclination $i \ldots \ldots \ldots$ & $11^{\circ \mathrm{d}}$ \\
\hline 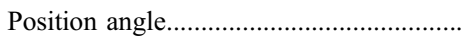 & $100^{\circ \mathrm{d}}$ \\
\hline 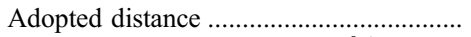 & $3.3 \mathrm{Mpc}^{\mathrm{e}}$ \\
\hline Corrected total $B$ magnitude $B_{T}^{0, i} \ldots \ldots \ldots \ldots$ & $10.93^{\mathrm{f}}$ \\
\hline Apparent blue magnitude $m_{B} \ldots \ldots \ldots \ldots \ldots \ldots$ & $11.21^{\mathrm{f}}$ \\
\hline Total blue luminosity $\left(M_{\odot}=5.43\right) \ldots \ldots \ldots$ & $6.8 \times 10^{8} L_{B_{\odot}}$ \\
\hline \multicolumn{2}{|c|}{$\begin{array}{l}\text { Note.-Units of right ascension are hours, minutes, and } \\
\text { seconds, and units of declination are degrees, arcminutes, and } \\
\text { arcseconds. } \\
\text { a From NED. } \\
\text { b From del Río (2004). } \\
\text { c From Baggett et al. (1998). } \\
\text { d Values for } r<200^{\prime \prime} . \\
\text { e From Tonry et al. (2001). } \\
\text { f From de Vaucouleurs et al. (1991). }\end{array}$} \\
\hline
\end{tabular}

It contains an appreciable amount of atomic (Baars \& Wendker 1976) and molecular gas (Wiklind \& Henkel 1990), a prominent, semicircular dust lane encircling the nucleus (Barbon et al. 1982), and various bright UV point sources (Maoz et al. 1998). It was classified a LINER by Schmidt et al. (1990). Morgan (1958) classified this galaxy as $E_{\mathrm{pec}}$, because of the dust lane. Humason et al. (1956) and Sandage (1961) classified it as an $\mathrm{S}_{3}$. Based on photographic surface photometry, Barbon et al. (1982) show that the object consists of bulge, lens, and exponential disk components, characteristic of a lenticular galaxy. This easily rules out a classification as (dwarf) elliptical, as several authors have proposed.

For many years the distance to NGC 404 was basically an unknown quantity. Estimates ranged from 1.5 (Baars \& Wendker 1976 ) to $10 \mathrm{Mpc}$ (Wiklind \& Henkel 1990). It is only recently that a firm distance to this object has been established. Tonry et al. (2001) used the surface brightness fluctuation method to come up with a distance of $3.3 \mathrm{Mpc}$. This was confirmed independently by Karachentsev et al. (2002), who used the tip of the red giant branch and determined a distance of $3.15 \pm 0.32 \mathrm{Mpc}$.

Although NGC 404 is listed as a member of the loose group LGG 011 (García 1993), it is unlikely to be physically associated with the rest of the group as at $3.3 \mathrm{Mpc}$ (Tonry et al. 2001) the nearest member, NGC 598 (M33), is located at 2.6 Mpc. Rather, NGC 404 is likely the nearest isolated S0 galaxy, especially as there are no other known galaxies within $1.1 \mathrm{Mpc}$ of it (Karachentsev et al. 2002). It is interesting to note that the field is only $2^{\circ}$ away from the center of the Piscis Cluster of galaxies $\left(\alpha=01^{\mathrm{h}} 10^{\mathrm{m}}, \delta=33^{\circ} 4\right)$. A summary of optical data on NGC 404 is given in Table 1.

\section{OBSERVATIONS}

NGC 404 was observed with the NRAO Very Large Array $(\text { VLA })^{2}$ on 1996 April 1 (C configuration) and 1996 July 16

\footnotetext{
${ }^{2}$ The National Radio Astronomy Observatory (NRAO) is a facility of the National Science Foundation operated under cooperative agreement by Associated Universities, Inc.
}

TABLE 2

VLA OBSERVING Log

\begin{tabular}{ccc}
\hline \hline Parameter & Configuration C & Configuration D \\
\hline Date of observation................... & 1996 Apr 1 & 1996 Jul 16 \\
Time on source (minutes)........... & 132.5 & 54 \\
Total bandwidth (MHz)............. & 1.56 & 1.56 \\
Number of channels................. & 128 & 128 \\
Velocity resolution $\left(\mathrm{km} \mathrm{s}^{-1}\right) \ldots \ldots \ldots$. & 2.57 & 2.57 \\
Central velocity $\left(\mathrm{km} \mathrm{s}^{-1}\right) \ldots \ldots \ldots \ldots$. & -57 & -57 \\
\hline
\end{tabular}

(D configuration) in the $21 \mathrm{~cm}$ line of neutral hydrogen (H I). In $\mathrm{C}$ configuration (with baselines between 100 and $3000 \mathrm{~m}$, or 0.5 to $15 \mathrm{k} \lambda$ ) the object was observed for 132 minutes; in D configuration (with baselines between 20 and $1000 \mathrm{~m}$ or 0.1 to $5 \mathrm{k} \lambda$ ), it was observed for 54 minutes. The absolute flux calibration was determined by observing $3 \mathrm{C} 48$, which was assigned a flux density of $15.99 \mathrm{Jy}$. This calibrator was also used to derive the complex bandpass correction. A nearby calibrator $(0116+319)$ was used as secondary amplitude and phase calibrator, and its flux was determined to be $2.635 \pm 0.005 \mathrm{Jy}$. Details about the observations can be found in Table 2. The data were edited and calibrated separately for each array with the AIPS package ${ }^{3}$ using standard reduction techniques.

The systemic velocity of NGC 404 is approximately $-54 \mathrm{~km}$ $\mathrm{s}^{-1}$ at a Galactic latitude of $-27^{\circ} .01$, hence some of the channels were confused with Galactic emission (in particular between -52 and $-46 \mathrm{~km} \mathrm{~s}^{-1}$ ). Since Galactic emission shows its presence on predominantly large scales, we were able to remove the most prominent emission features by blanking the $u$-v data out to $0.5 \mathrm{k} \lambda$ in these channels (this affects only D configuration data).

The $u-v$ data were examined, and bad data points caused by either interference or cross talk between antennae were removed, after which the data were calibrated. We Fourier transformed our C- and D-configuration observations separately to assess their quality. Finally, we combined both data cubes to form a single data set, which was used for further analysis. The original bandwidth of the data was $1.56 \mathrm{MHz}$; for the rest of this paper, we use a $0.69 \mathrm{MHz}$ wide bandwidth around the spectral region of interest. The data have a velocity resolution, after online Hanning smoothing, of $12.2 \mathrm{kHz}$ $\left(2.57 \mathrm{~km} \mathrm{~s}^{-1}\right)$.

Channels with velocities higher than $16 \mathrm{~km} \mathrm{~s}^{-1}$ and lower than $-128 \mathrm{~km} \mathrm{~s}^{-1}$ were determined to be line-free. A total of 20 channels on either side of the main profile containing no line emission were averaged to form a "dirty" continuum image, again avoiding the edge channels of the bandpass. The average continuum level was subtracted from the $u-v$ data.

\section{H I SPECTRAL CHANNEL MAPS}

High $\left(\sim 15^{\prime \prime}\right)$ resolution cubes were obtained using the task imagr with the parameter robust $=-1$ (where robust $=-5$ corresponds to uniform weighting and robust $=5$ to natural weighting). With this value we obtain the best compromise between the smallest beam (almost identical to "uniform") and the lowest noise (almost identical to "natural"). Cleaning was applied down to a flux level of 2 times the average rms

\footnotetext{
${ }^{3}$ The Astronomical Image Processing System (AIPS) has been developed by the NRAO.
} 


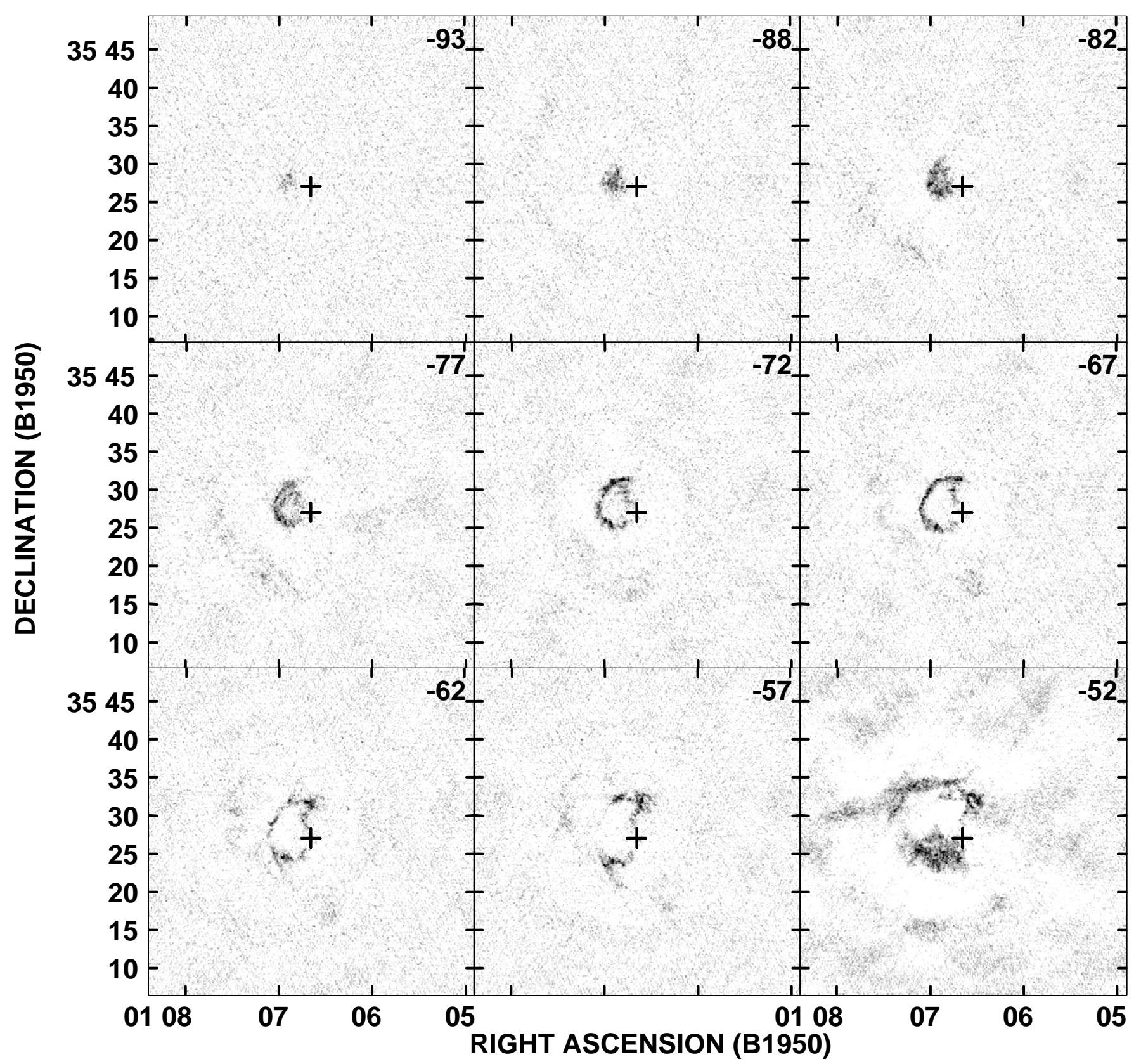

Fig. 1.-H I line channel maps of NGC 404 after subtraction of the continuum emission and cleaning. The spatial resolution (HPBW) is 15 ".2 $\times 14$."4. In order to save space, each panel is the average of two neighboring channels. The heliocentric velocity of each channel has been indicated in kilometers per second. The hatched circle in the lower right corner of the top left panel shows the beam size. The gray scale runs from 0 to $5 \mathrm{mJy} \mathrm{km} \mathrm{s}^{-1}$. The cross indicates the optical and radio continuum center. Coordinates are B1950.0 equinox.

noise in the maps and in an area large enough to include all the prominent emission. Figure 1 shows the resulting channel maps (before blanking). Notice that most traces of contamination by Galactic emission have been successfully removed. To save space, we display the average of two consecutive channels in each panel over the velocity range in which line emission is present. The heliocentric radial velocities (in kilometers per second) are plotted in the upper right-hand corner of each panel. The beam size $\left(15^{\prime \prime} .2 \times 14^{\prime \prime} 4\right)$ is indicated in the top left-hand panel, left bottom corner (the beam size is so small that it cannot be properly reproduced in our maps). Already at first glance we can see quite strong emission (for an S0 galaxy). The resulting high-esolution cubes $(512 \times$ 512 pixels $\times 57$ channels at $15^{\prime \prime} 2 \times 144^{\prime \prime} 4$ ) were smoothed to a spatial resolution of $25^{\prime \prime} \times 25^{\prime \prime}(\mathrm{HPBW})$ in order to bring out the faint, extended structures (referred to henceforth as lowresolution cubes). Table 3 lists the characteristics of the resulting data cubes.

\section{GLOBAL H I CONTENT}

The global $\mathrm{H}$ I profile was obtained by integration over the individual high-resolution channel maps (note that this can be safely done as all continuum emission was subtracted in the $u$-v plane). Before the summation, the flux at each position was corrected for attenuation by the primary beam of the array. The resulting global profile is shown in Figure 2. From the global profile we derive a total $\mathrm{H}$ I flux of $59.3 \pm$ $1.7 \mathrm{Jy} \mathrm{km} \mathrm{s}{ }^{-1}$, a systemic velocity of $-53.9 \mathrm{~km} \mathrm{~s}^{-1}$, and a 


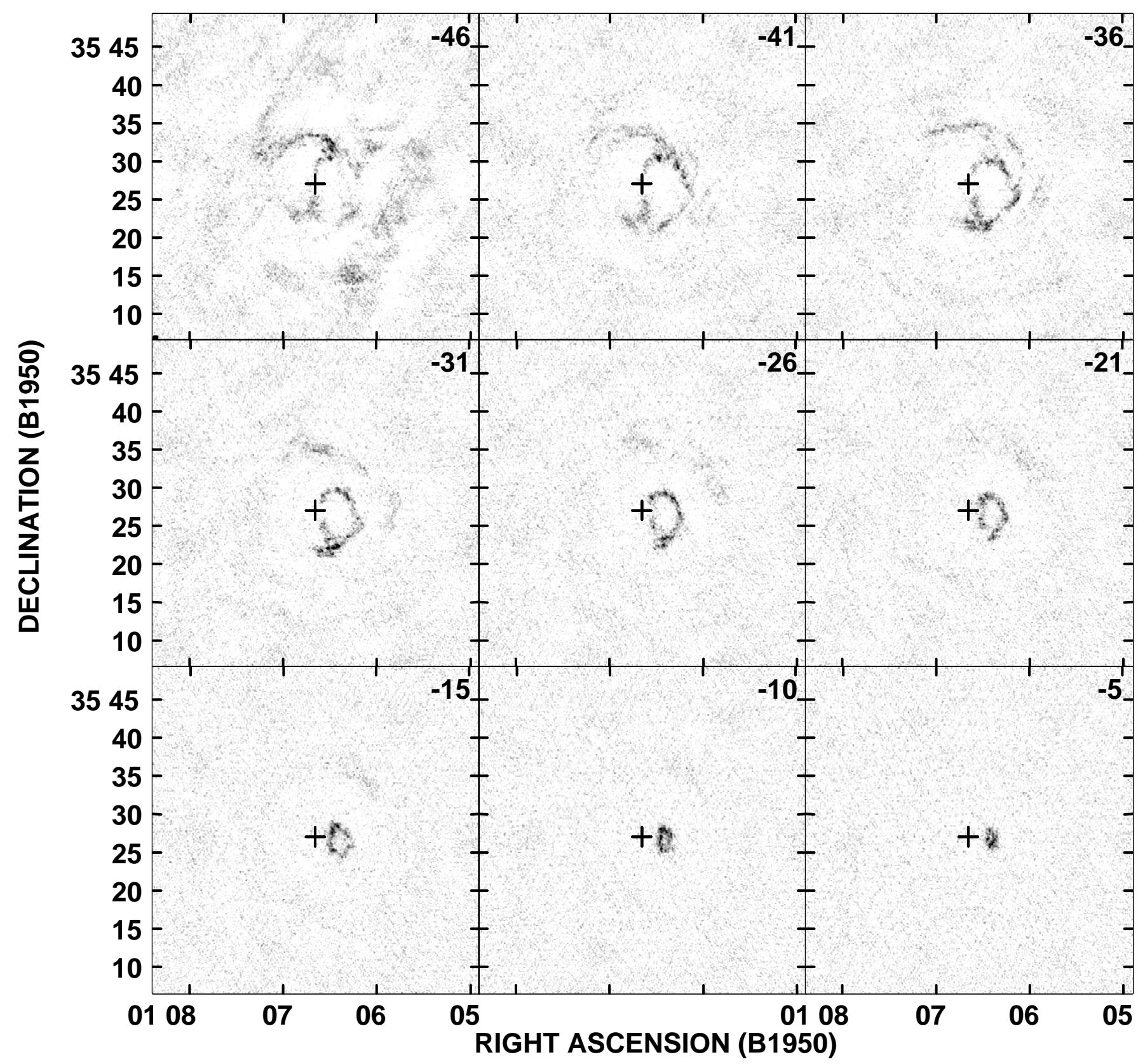

Fig. 1.—Continued

TABLE 3

Characteristics of the Data Cubes

\begin{tabular}{|c|c|}
\hline Parameter & Value \\
\hline \multicolumn{2}{|l|}{ High Resolution } \\
\hline 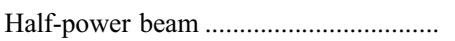 & $15^{\prime \prime} 2 \times 14^{\prime \prime} 4$ \\
\hline 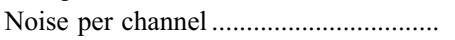 & $1.1 \mathrm{mJy}^{\text {beam }}{ }^{-1}$ \\
\hline Conversion factor $\left(1 \mathrm{mJy}\right.$ beam $\left.^{-1}\right) \ldots . .$. & $2.7 \mathrm{~K}$ \\
\hline \multicolumn{2}{|l|}{ Low Resolution } \\
\hline Half-power beam ........ & $25^{\prime \prime} \times 25^{\prime \prime}$ \\
\hline 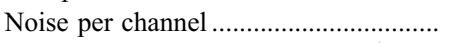 & $0.65 \mathrm{mJy}$ beam $^{-1}$ \\
\hline Conversion factor $\left(1 \mathrm{mJy}\right.$ beam $\left.^{-1}\right) \ldots \ldots$. & $1.0 \mathrm{~K}$ \\
\hline
\end{tabular}

global $\mathrm{H}$ I velocity profile width at $20 \%$ and $50 \%$ of the maximum of 85 and $65 \mathrm{~km} \mathrm{~s}^{-1}$ (not corrected for inclination, instrumental resolution, turbulent or $z$-direction gas motion; the error is of order one channel width or $2.5 \mathrm{~km} \mathrm{~s}^{-1}$ ).

In order to derive the correct $\mathrm{H}$ I flux in each of the channel maps, one has to keep in mind that the determination of $\mathrm{H} \mathrm{I}_{\mathrm{I}}$ fluxes of extended emission in interferometric maps is not straightforward. As explained by Jörsäter \& van Moorsel (1995), fully cleaned maps do not exist, and any cleaned map consists of the sum of two maps: one containing the restored clean components and the other, the residual map. In the former, the unit is janskys per clean beam area, and in the latter janskys per dirty beam area. Following Walter \& Brinks (1999), we used our cleaned data cube of NGC 404 in order to calculate the real flux of our channels. We calculate this real flux $(G)$ using $G=(D C) /(D-R)$, where $C$ is the cleaned 


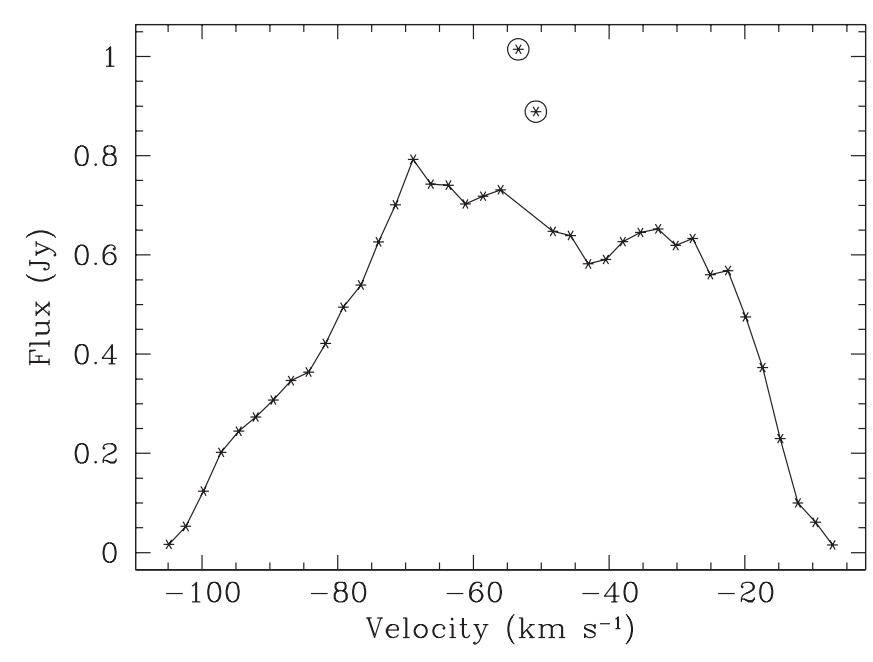

FIG. 2.-Global H i profile. The two encircled points represent values that are contaminated by Galactic emission that cannot be completely removed from the data cube.

flux, and $R$ and $D$ are the "erroneous" residual flux and the "erroneous" dirty flux over the same area of the channel map, respectively. For a full discussion on this topic, the reader is referred to the appendix in Jörsäter \& van Moorsel (1995). We followed this procedure for a selected sample of channels and compared the results with our estimates for the real flux, which were obtained by a direct, deep clean. The agreement is to within a few percent, indicating that using our cleaned maps we get values that are very close to the real flux.

Our integrated $\mathrm{H}$ I flux is in fair agreement with that estimated by Baars \& Wendker (1976) using the Effelsberg $100 \mathrm{~m}$ telescope who arrived at a value of $42 \mathrm{Jy} \mathrm{km} \mathrm{s}^{-1}$. It should be noted, however, that the latter value was reported as being highly uncertain because of contamination of the single dish spectra by foreground emission from the Galaxy and could be off by up to $25 \%$.

Using

$$
\begin{aligned}
& M_{\mathrm{HI}}\left(M_{\odot}\right)= 2.356 \times 10^{5} D^{2}(\mathrm{Mpc}) \\
& \times \int F_{i} d v\left(\mathrm{Jy} \mathrm{km} \mathrm{s}^{-1}\right),
\end{aligned}
$$

we find a total $\mathrm{H}$ I mass of $M_{\mathrm{HI}}=1.52 \times 10^{8} M_{\odot}$, which is an order of magnitude less than the mean mass found by van Driel \& van Woerden (1991) and references therein for their sample of $\mathrm{S} 0$ galaxies. The mean $\mathrm{H}$ I mass for $14 \mathrm{~S} 0$ galaxies is $\sim 2.0 \times 10^{9} M_{\odot}$, using $H_{0}=75 \mathrm{~km} \mathrm{~s}^{-1} \mathrm{Mpc}^{-1}$. The $\mathrm{H}$ I mass-to-blue luminosity ratio, $M_{\mathrm{HI}} / L_{B}=0.22$, which is independent of distance, is, on the other hand, high for an S0 galaxy and much closer to that found for later type spirals (Roberts \& Haynes 1994).

A quick calculation shows that NGC 404 is underluminous for an S0 galaxy, as indicated by the Tully-Fisher (1977) relation for this object. Correcting the global profile measured at the $20 \%$ level for instrumental broadening and for turbulent and $z$-direction motions by $-12.6 \mathrm{~km} \mathrm{~s}^{-1}$ (following Richter \& Huchtmeier 1984) and assuming an inclination of $11^{\circ}$ (the value that we have found for the inner part of the disk), we estimate a peak to peak velocity for gas in the disk of $380 \mathrm{~km} \mathrm{~s}^{-1}$ or a circular velocity of $190 \mathrm{~km} \mathrm{~s}^{-1}$. The required $B$ luminosity in order to have NGC 404 fall on the Tully-Fisher curve is more than an order of magnitude higher

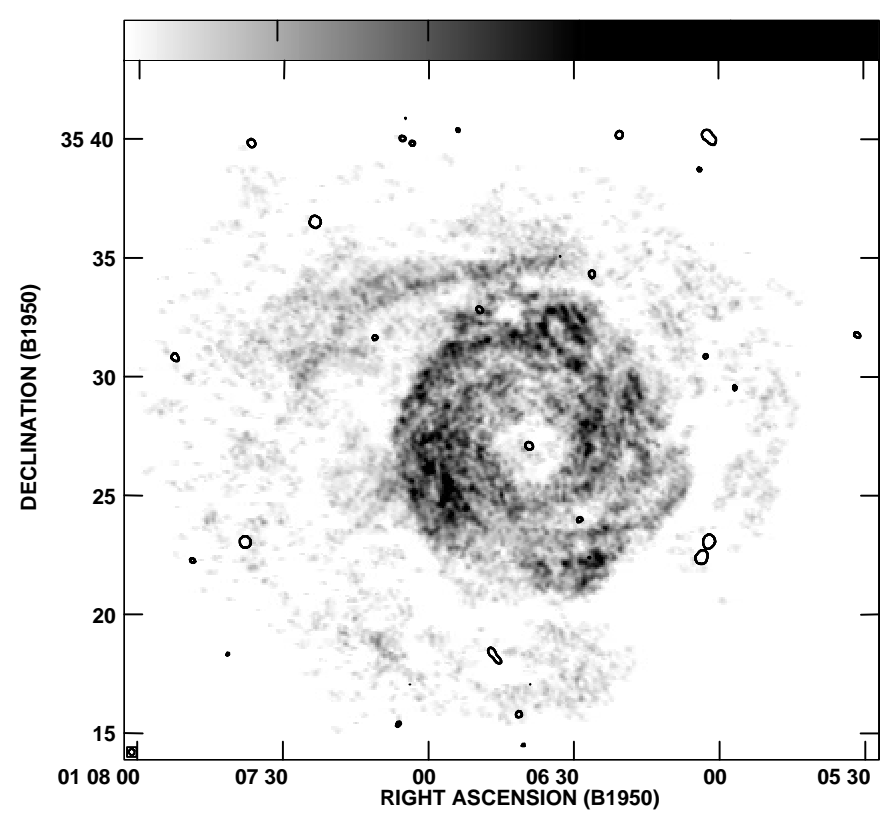

FIG. 3.- Integrated neutral hydrogen map of NGC 404. Intensities range from 0 to $300 \mathrm{~K} \mathrm{~km} \mathrm{~s}^{-1}$ or $5.5 \times 10^{20} \mathrm{~cm}^{-2}$. Continuum sources over $5 \sigma$ $(0.75 \mathrm{mJy})$ are superposed on the emission-line map.

( 2.5 to $3 \mathrm{mag}$ ) than the value we assumed here and would imply a distance of order $20 \mathrm{Mpc}$, which is completely ruled out by the distance determination of $3.3 \mathrm{Mpc}$ by Tonry et al. (2001) and Karachentsev et al. (2002).

\section{5. $\mathrm{H}$ I DISTRIBUTION AND VELOCITY FIELD}

After Fourier transforming the $u-v$ data, the resulting data cube was conditionally blanked, accepting as genuine all emission at levels above $2 \sigma \mathrm{rms}$ noise in the data cube smoothed to a resolution of $25^{\prime \prime}$ and requiring that the emission is seen in at least three consecutive channels. Maps of the $\mathrm{H}$ I column density distribution and velocity field were obtained by taking the zeroth and first moment of the spectra at each pixel along the velocity axis. This was done in both the high- and low-resolution cubes.

\subsection{H I Distribution}

The $\mathrm{H}_{\mathrm{I}}$ is distributed as a bright doughnut with an inner diameter of $\simeq 100^{\prime \prime}$ and an outer diameter of $\simeq 400^{\prime \prime}$ (see Fig. 3), that is, from $R \sim R_{25}$ to $R \sim 4 R_{25}$. At a considerably lower signal-to-noise ratio, an outer ring can be discerned out to twice that diameter. The ellipticity of that ring is higher than that of the disk, suggesting a different inclination. Our VLA H I data imply a mass of $M_{\mathrm{HI}} \simeq 1.14 \times 10^{8} M_{\odot}$ in the "galaxy" $\left(R<400^{\prime \prime}\right)$ and $M_{\mathrm{HI}} \simeq 0.38 \times 10^{8} M_{\odot}$ in the "annulus" $(R>$ $\left.400^{\prime \prime}\right)$, that is, roughly a distribution of $\frac{3}{4}$ of the total mass in the galaxy and $\frac{1}{4}$ in the outer ring. There is considerable finescale structure visible, but no large-scale pattern such as spiral arms. The doughnut is very nearly circular, suggesting an almost face-on orientation. This is corroborated by isophotal fits to the optical image. Barbon et al. (1982) derive values for the inclination and position angle as a function of radius, from $R \sim 2^{\prime \prime}$ to $\sim 88^{\prime \prime}$. The values they found vary from $158^{\circ}$ to $75^{\circ}$ in P.A. and from 0.238 to 0.062 in ellipticity. Wiklind \& Henkel (1990) use a constant value of $i=16^{\circ}$ and a position angle, P.A. $=100^{\circ}$.

The azimuthally averaged radial distribution of the $\mathrm{H}$ i surface density, corrected to face-on, $\sigma_{M_{\mathrm{HI}}}$, is shown in Figure 4 


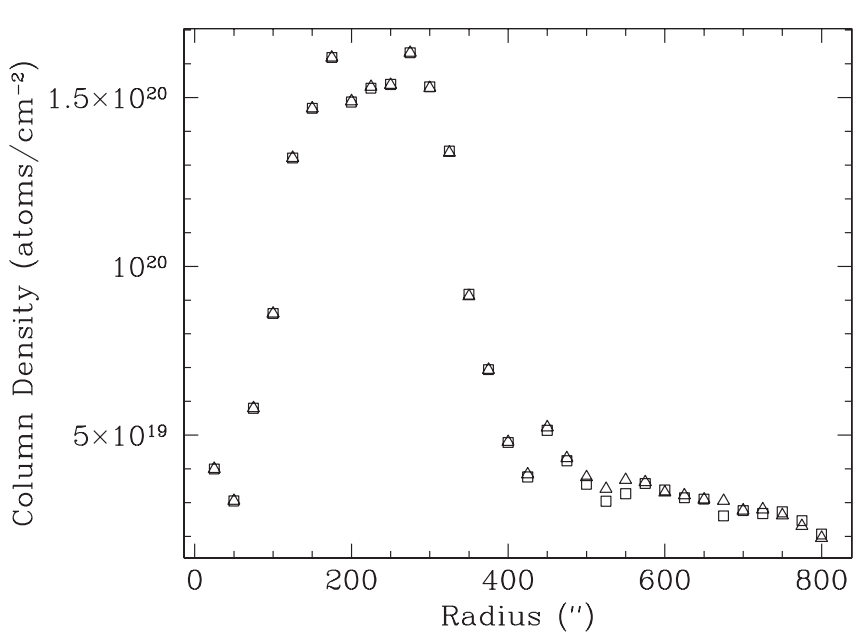

FIG. 4.-Mean column density as a function of radius, obtained by averaging in rings. In this graph one can clearly appreciate the doughnut. (Triangles: fits to the galaxy assuming a flat rotation curve; squares: fits assuming a Keplerian decline.)

for the full-resolution data. The profile was derived from the $\mathrm{H}$ I column distribution map by averaging in circular rings in the plane of the galaxy, centered at the position of the nuclear continuum source, with the task iring of GIPSY. ${ }^{4}$ The values for P.A. and $i$ are those derived based on the velocity field for a flat and a Keplerian rotation curve (see $\S 6$ for details). There are no significant differences, however, between the two sets of inclination angles in determining the $\mathrm{H}$ I surface density.

The surface-brightness versus radius profile cannot be adjusted to any of the distribution functions that usually characterize these profiles (Gaussian or modified Hubble profile). Neither corresponds to a truncated disk either since the decrease beyond $R=400^{\prime \prime}$ is much steeper than an exponential.

\subsection{Velocity Field}

A velocity field based on the smoothed, $25^{\prime \prime} \times 25^{\prime \prime}$ channel maps is presented in Figure 5(top). We note that the isovelocity contours are closed and that the velocities of the external annulus do not form a smooth continuation with those of the main body. Also, the line of nodes varies as a function of radius implying the presence of a warp. The projected radial velocities range from about -100 to about $-20 \mathrm{~km} \mathrm{~s}^{-1}$. The velocities of the outer ring fall within the same range as those of the main body, but the position angle shows a jump (see Fig. 6).

\section{ROTATION CURVE}

The velocity field in the inner region $\left(<400^{\prime \prime}\right)$ is sufficiently regular to permit the determination of a rotation curve for which we used a tilted-ring model as implemented by the task rotcur in the GIPSY package (Rogstad et al. 1974; Begeman 1989).

The model assumes circular symmetry in each of a set of concentric, but not necessarily coplanar, rings centered on the nucleus. The projected circular velocity $V(r, \theta, i)$ (which is based on the observations) is related to the real velocity $V_{c}(r)$, assuming circular orbits of the gas, via

$$
V(r, \theta, i)=V_{\mathrm{sys}}+V_{c}(r) \sin (i) \cos (\theta-\text { P.A. })
$$

\footnotetext{
${ }^{4}$ The Groningen Image Processing System (GIPSY) is distributed by the Kapteyn Astronomical Institute, Groningen, Netherlands.
}

where $V_{\text {sys }}$ is the systemic velocity, $i$ is the inclination angle, P.A. is the position angle of the receding major axis (the line of nodes), and $\theta-$ P.A. is the angle measured with respect to the receding major axis of the object.

To find a set of these parameters that best represent the observed velocity field, rotcur performs nonlinear least-squares fits to the observed radial velocities in a set of concentric elliptical annuli in the plane of the sky, each one beamwidth (25" after smoothing, Fig. 5) wide along the major axis. Points on either side of the major axis are included up to $\theta=60^{\circ}$ (i.e., we excluded a sector around the minor axis). Using best guesses for the systemic velocity $\left(V_{\text {sys }}\right)$ and the kinematical center of the object, fitted values for $i$, P.A., and an approximate rotation curve were calculated. Once fits for $i$ and P.A. as a function of radius were found, these values were held fixed and $V_{\text {sys }}$ and the central position were fitted. We repeated this procedure a couple of times until consistent values for the central position and $V_{\text {sys }}$ were arrived at. The systemic velocity of $-55.4 \mathrm{~km} \mathrm{~s}^{-1}$ and the kinematical center, $\alpha_{\mathrm{B} 1950.0}=01^{\mathrm{h}} 06^{\mathrm{m}} 39 \mathrm{~s} .40$ and $\delta_{\mathrm{B} 1950.0}=35^{\circ} 27^{\prime} 5^{\prime \prime}$, are found to be in good agreement with published values: based on observations of ${ }^{12} \mathrm{CO}(1 \rightarrow 0)$, Sage $(1990)$ found $V_{\text {sys }}=$ $-58 \mathrm{~km} \mathrm{~s}^{-1}$; Wiklind \& Henkel (1990) derived $V_{\mathrm{sys}}=$ $-56 \mathrm{~km} \mathrm{~s}^{-1}$.

We then proceeded to minimize the dispersion of $V_{c}(r), i$, and P.A. for each ring. It should be noted that the solution for $V_{c}(r)$ and $i$ are coupled through the factor $V_{c}(r) \sin (i)$ in equation (2) and that this coupling is very strong for low inclinations. In the case of NGC 404 we decided to adopt an inclination of $i=11^{\circ}$ near a radius of $200^{\prime \prime}$, close to the location of the peak of the observed rotation curve. This inclination is based on an intensity-weighted average of the ellipticity as a function of radius of the $\mathrm{H}$ I doughnut. This inclination, being derived under the assumption that the $\mathrm{H}$ i is distributed in a thin disk, is likely a better approximation of the true value than the inclination quoted by Barbon et al. (1982). With this choice of inclination, the observed peak velocity translates to an intrinsic peak rotational velocity of about $200 \mathrm{~km} \mathrm{~s}^{-1}$ at a radius of $200^{\prime \prime} .^{5}$

Not being able to independently fit $V_{c}(r)$ and $i$, we instead considered two extreme solutions, the first one being a Keplerian decline (model K). This model is inspired by the observed radial velocity as a function of radius, which follows in its outer parts an $r^{-1 / 2}$ curve. Fits to the receding and approaching side of an $r^{-1 / 2}$ decline agree to within $1 \sigma$. We followed Shu et al. (1971) and constructed a Keplerian rotation curve, based on the sum of an inhomogeneous spheroid and a Toomre disk with $n_{\mathrm{T}}=3$ scaled to match the peak velocity of our data.

With the rotational velocity thus fixed, we reran rotcur and for each model we left only as free parameters to be fitted P.A. and $i$. In Figure 5 (bottom) we show the residual map after subtracting a fit to a Keplerian declining rotation curve. Figure 7 shows the same observed velocity field as presented in Figure 5 and, using the same color scheme, the resulting model velocity field. From these figures the excellent agreement between model and observations can be appreciated.

The second solution we adopted was for the rotation curve to remain flat (model F) after reaching a peak rotational

\footnotetext{
5 An inclination of $i=11^{\circ}$ is a lower limit. A more relaxed value would be $i=16^{\circ}$, as used by Wiklind \& Henkel (1989), which results in a peak velocity corrected for inclination a factor of 1.4 lower, at $140 \mathrm{~km} \mathrm{~s}^{-1}$, and dynamical masses a factor of 2 lower.
} 

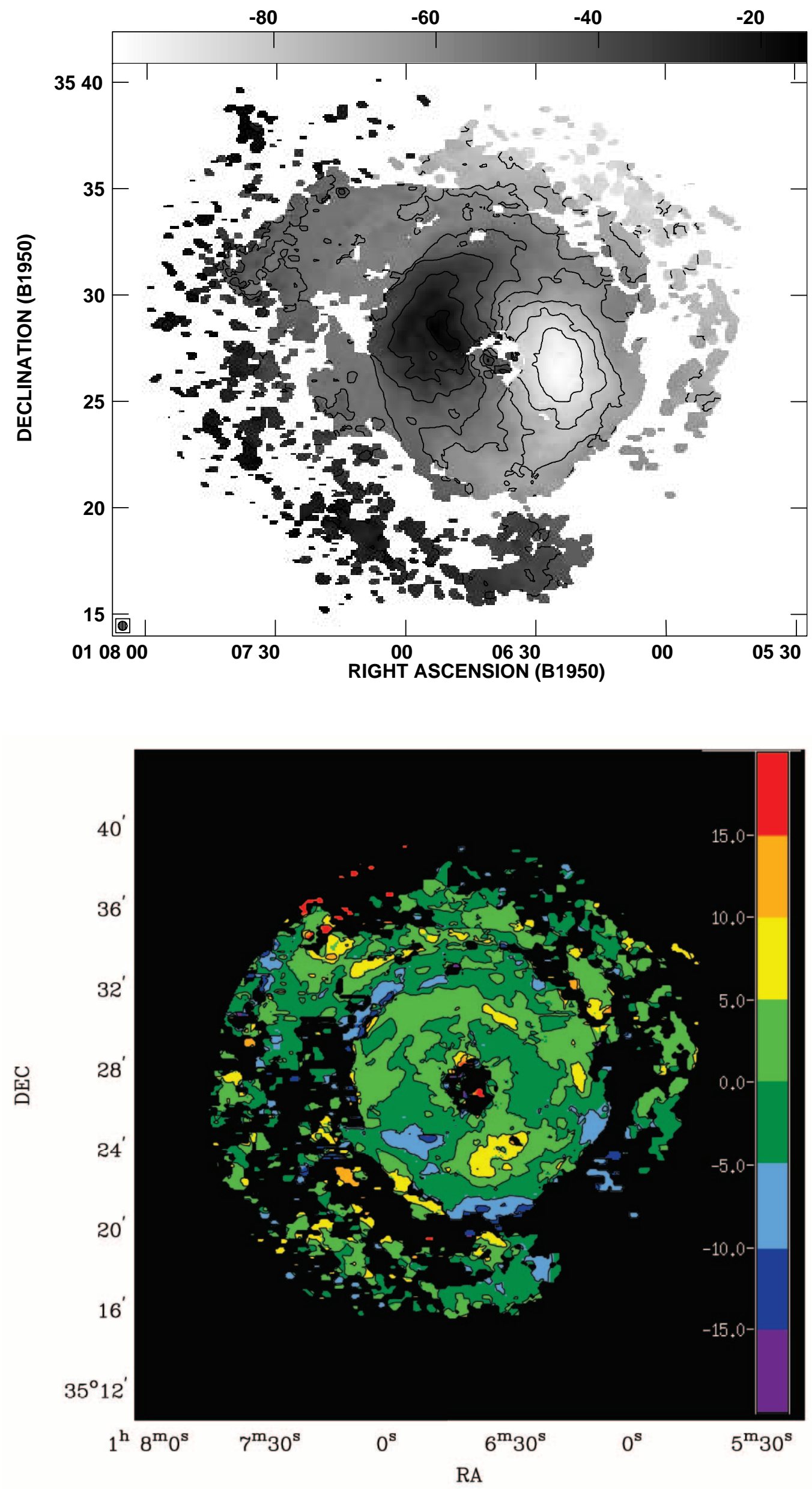

FIG. 5.-Top: Velocity map. The observed radial velocities range from -100 to $-20 \mathrm{~km} \mathrm{~s}^{-1}$, the western half being the receding side. Bottom: Residual map obtained after subtracting a Keplerian model from the data. Contours range from -10 to $10 \mathrm{~km} \mathrm{~s}^{-1}$ in steps of $5 \mathrm{~km} \mathrm{~s}^{-1}$. 


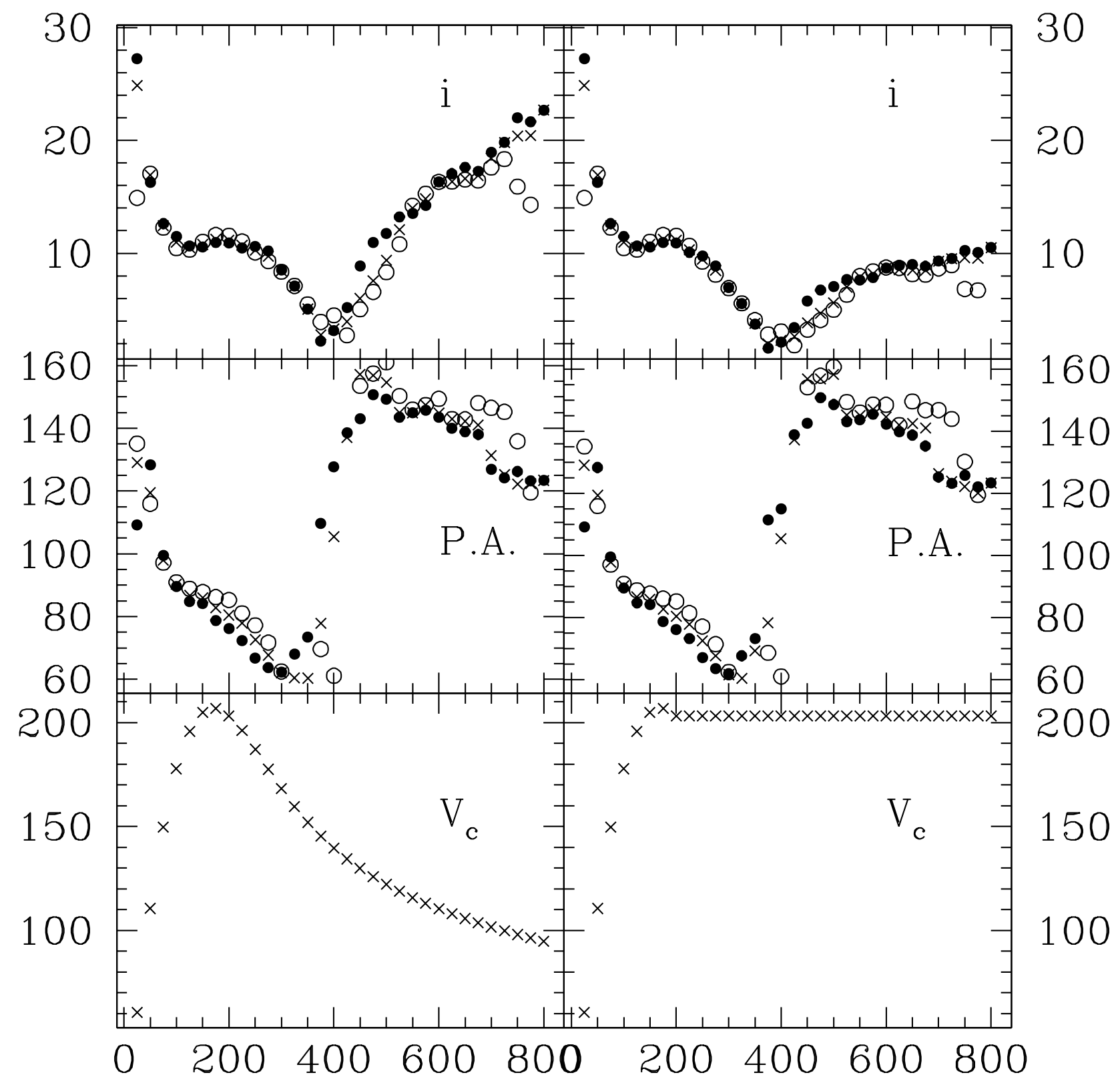

FIG. 6.-Tilted-ring model fits to the velocity field of NGC 404 showing solutions for a Keplerian (left) and flat (right) rotation curve. The axes are labeled as follows: P.A. and $i$ are in degrees, $V_{c}$ is in kilometers per second, and the radial distance from the center is in arcseconds. Crosses represent the result of the average for both sides. Open circles correspond to the fit of the receding and filled circles to that of the approaching side, respectively.

velocity of $200 \mathrm{~km} \mathrm{~s}^{-1}$ at a radius of $200^{\prime \prime}$. Graphical representations of this model and corresponding residual velocity maps are indistinguishable from model $\mathrm{K}$.

The results of the model fits, restricted as described above, are shown in Figure 6. In this figure the values for the receding and approaching sides, as well as the average over both sides, are plotted. The first thing we note is that for both models the position angles share practically the same values, indicating that this parameter is insensitive to either choice of rotation curve and probably reflects the true run of position angle with radius. The two first points $\left(R \leq 50^{\prime \prime}\right)$ are not significant as they fall within the $\mathrm{H}$ I hole (see Fig. 8), where there is scant gas and the fit can take almost any value. From $R>50^{\prime \prime}$ to $\sim 350^{\prime \prime}$, the value for the P.A. declines monotonically and almost linearly from $100^{\circ}$ to $60^{\circ}$. This is depicted in an alternative manner in Figure 9. There is a discontinuity at $R \sim 400^{\prime \prime}$ (close to $4 R_{25}$ ). It is exactly around this radius that we reach the edge of the main body of the galaxy and that we see the transition to the annulus. Finally, the annulus shows the same tendency, but less pronounced by a factor of 2 , with the P.A. decreasing from $160^{\circ}$ to $120^{\circ}$.

The behavior of P.A. being so similar in both models, the most significant differences then have to be a function of $i$, and mainly for $R>200^{\prime \prime}$, where the functional behavior of both rotation curves starts to diverge. This is indeed what we find. Within the errors, the inclination for the doughnut is constant, differences as small as $1^{\circ}$ being sufficient to mask the difference between the $\mathrm{K}$ and $\mathrm{F}$ models. Beyond $R \sim 500^{\prime \prime}$, the inclination in the case of the $\mathrm{K}$ model has to increase, whereas in the case of the F model it remains roughly constant. Figure 10 is an attempt to visualize the shape and extent of the implied 

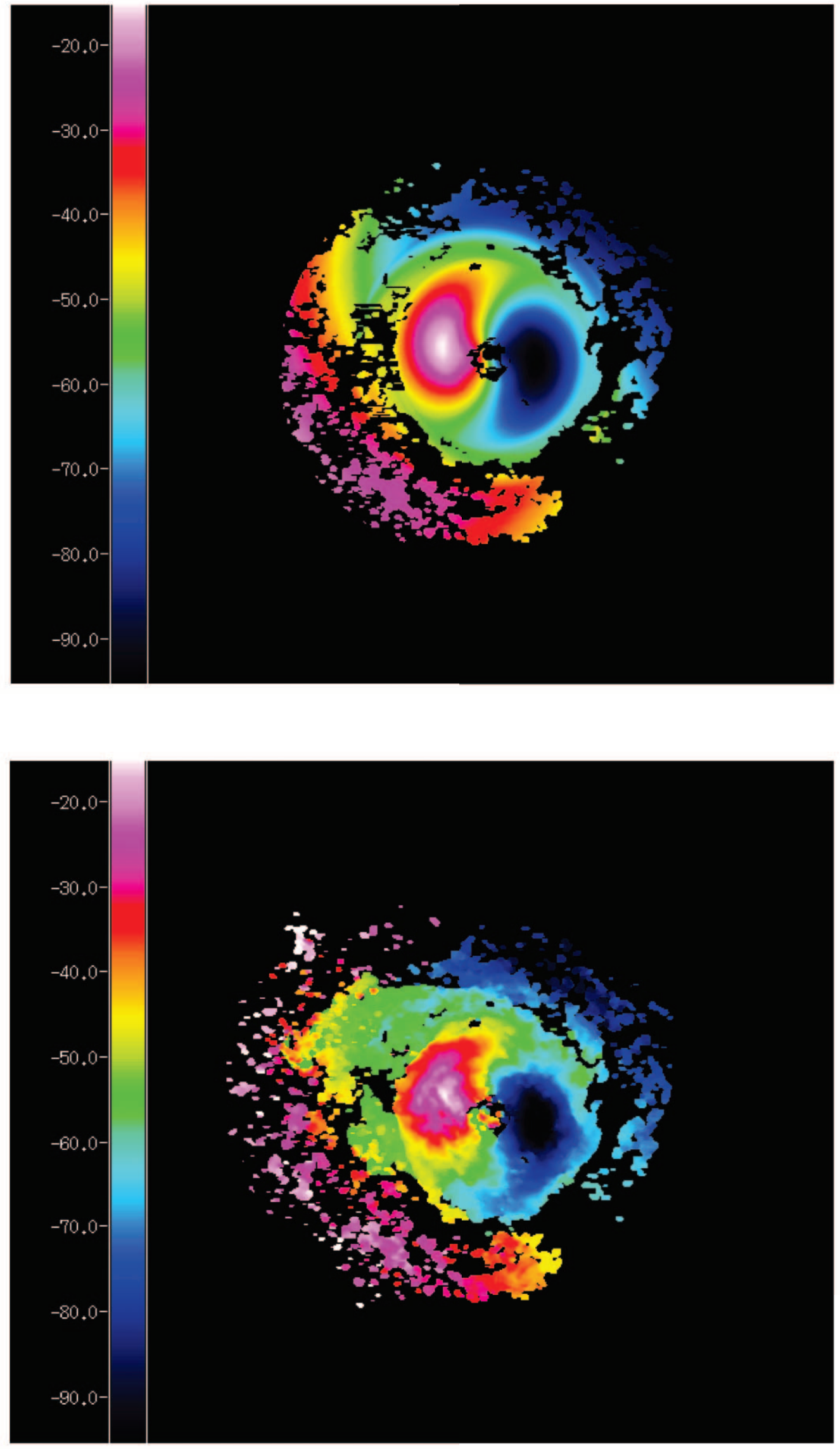

FIG. 7. - Top: Model velocity field based on a rotation curve exhibiting a Keplerian decline beyond a radius of $3.2 \mathrm{kpc}$. Bottom: Observed velocity field. The color scheme is the same for both panels. 

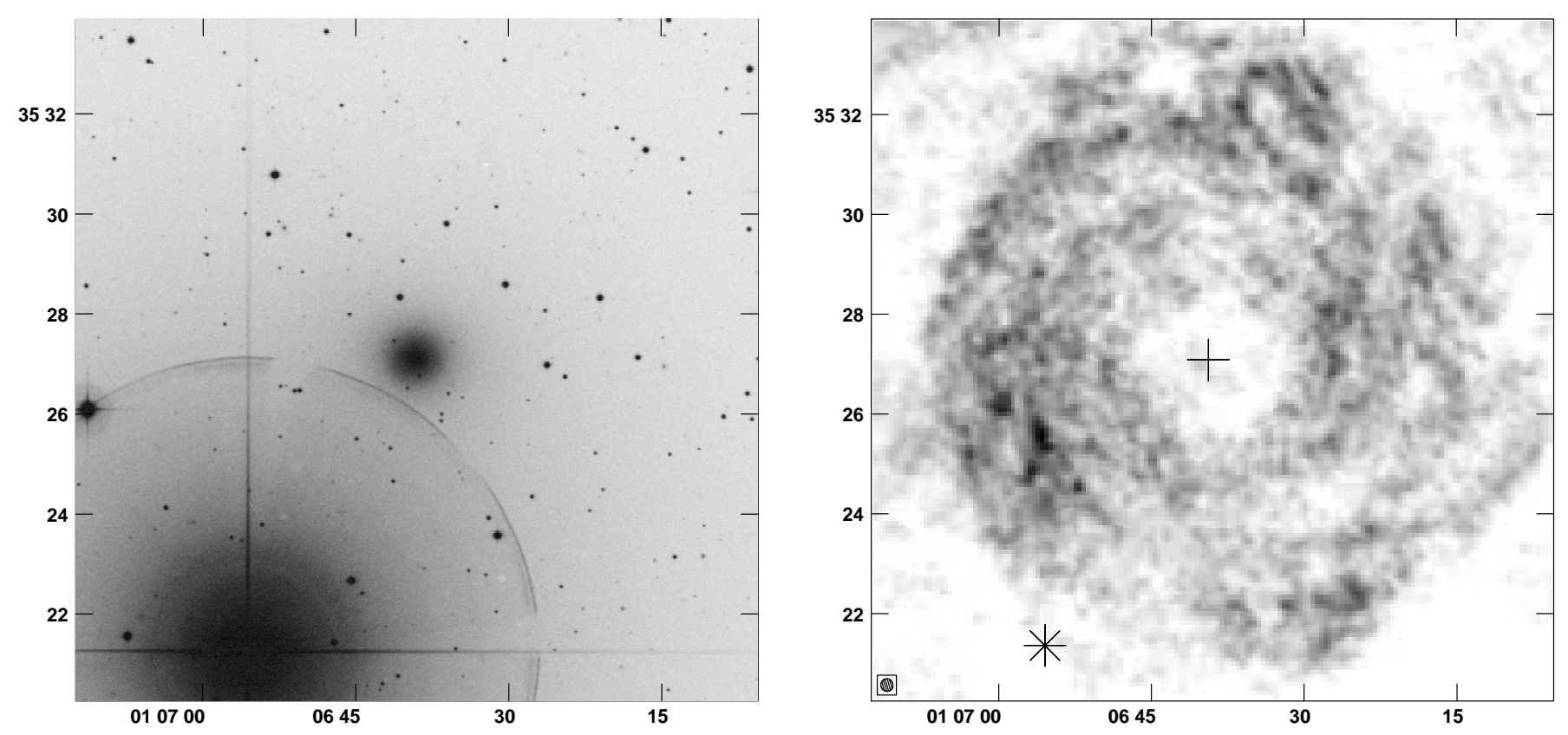

FIG. 8.-Display of an optical image taken from the DSS (left) and at the same scale, the H I distribution (right). The bright star object to the southeast of NGC 404 in the DSS image is $\beta$ And. The cross indicates the center of NGC 404; the asterisk represents the location of $\beta$ And.

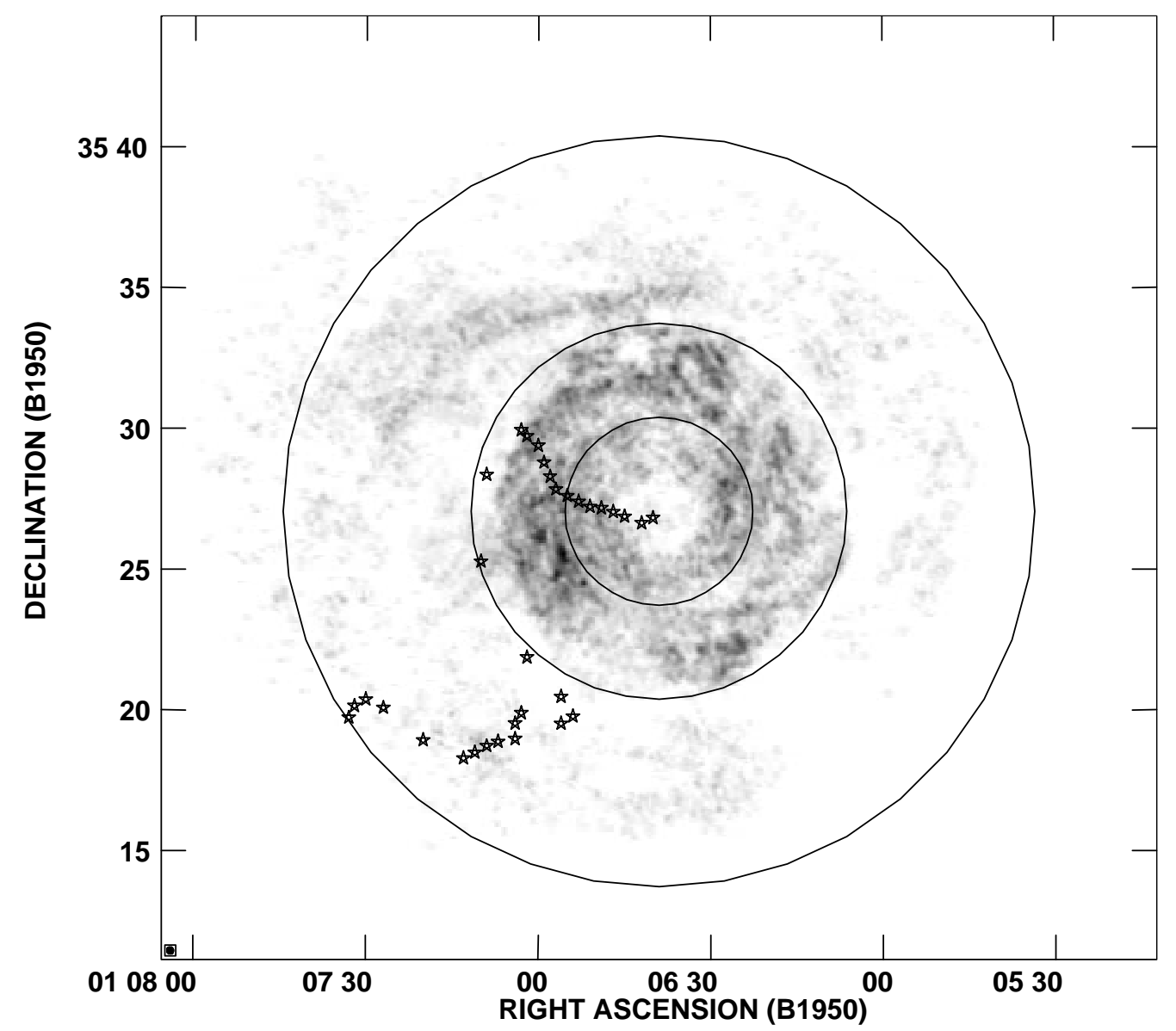

FIG. 9.-Direction of the P.A. drawn as five-pointed stars. The circles represent, from the innermost to the outermost: $R=200^{\prime \prime}$, where the rotation curve begins to decline $\left(\approx 2 R_{25}\right), R=400^{\prime \prime}$, which marks the outer rim of the doughnut, and $R=800^{\prime \prime}$, the last point of our velocity model. It can be seen that the P.A. is almost constant within $R_{25}\left(\sim 200^{\prime \prime}\right)$, at which radius the warp sets in. 


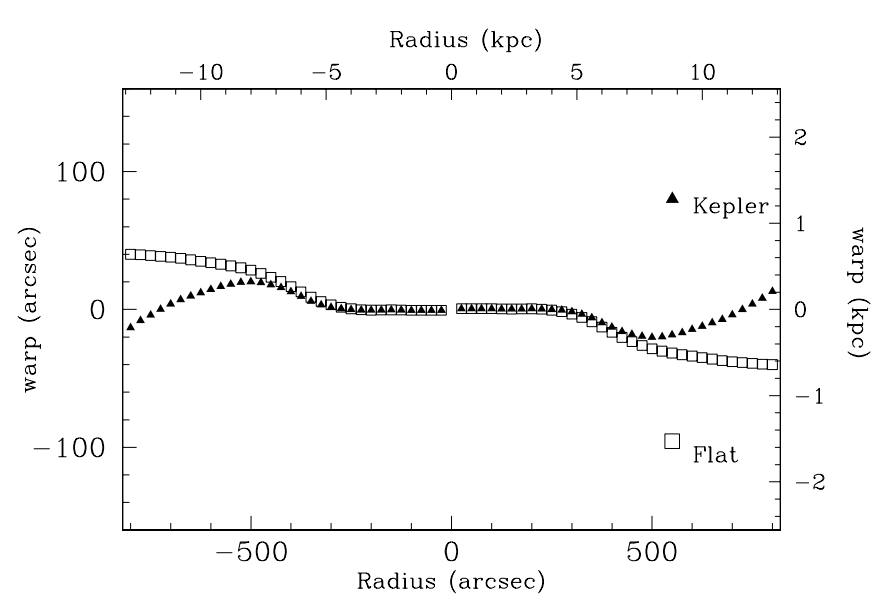

FIG. $10 a$

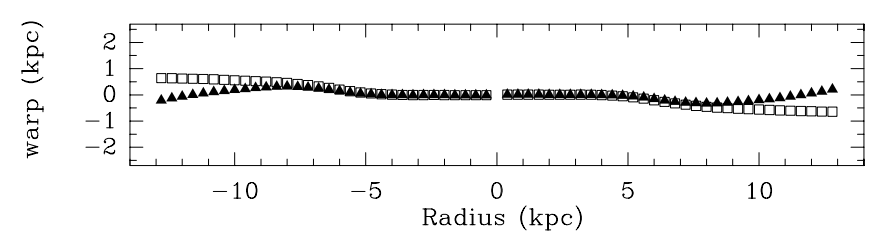

FIG. $10 b$

FIG. 10.-(a) Schematic view of both warps (for flat and Keplerian rotation curves) as seen from an edge-on perspective, in the plane of the galaxy. The $y$-axis has been enlarged to better visualize the differences between both warps. (b) As above, but with both axes having the same scale to better visualize the amplitude of the warp against the size (in $\mathrm{H} \mathrm{I}$ ) of the galaxy.

warp, both for a Keplerian decline as well as for a flat rotation curve.

\section{THE MASS OF NGC 404}

We can now proceed to derive the mass within the last measured point for both the $\mathrm{K}$ and $\mathrm{F}$ model fits to the velocity field. In the case of a Keplerian decline and assuming for simplicity a spherical mass model $\left(M_{T}=2.326 \times 10^{5} V_{\text {rot }}^{2} R\right.$, [ $M_{\mathrm{T}}$ in $M_{\odot}, V_{\text {rot }}$ in $\mathrm{km} \mathrm{s}^{-1}$ and $R$ in kiloparsecs]), we derive a total mass $M_{T} \approx 3 \times 10^{10} M_{\odot}$. This is assuming the peak rotational velocity of $200 \mathrm{~km} \mathrm{~s}^{-1}$ is reached at $200^{\prime \prime}\left(\sim 2 R_{25}\right)$ after which point the rotation curve falls off. In that case the global $M / L_{B}$ ratio is $\sim 44 M_{\odot} / L_{\odot}$. This is quite a high value and a factor of 1.5 higher than the already substantial values encountered by van Driel \& van Woerden (1991).

If we now consider a flat rotation curve, taking as the last measured point a value of $\sim 800^{\prime \prime}$, the mass turns out to be 4 times higher, $\sim 12 \times 10^{10} M_{\odot}$, and the mass-luminosity ratio would reach a staggering $\sim 175 M_{\odot} / L_{\odot}$.

Although the $M / L_{B}$ values are high, NGC 404 cannot be considered a massive galaxy. If we take the total mass enclosed within $R_{25}\left(R=1.65 \mathrm{kpc}\right.$ and $\left.V=160 \mathrm{~km} \mathrm{~s}^{-1}\right)$, we find $M_{T}=0.98 \times 10^{10} M_{\odot}$, less than $10 \%$ of the mean mass encountered within the same radius in the galaxies making up the sample of van Driel \& van Woerden (1991). In that case the mass-to-light ratio is $14.4 M_{\odot} / L_{\odot}$. Although this is still high, it is more in line with that found in early-type galaxies.

\section{CONTINUUM $1.4 \mathrm{GHz}$}

To check for the possible presence of low-level continuum emission, we extracted 34 channels at either side of the combined $u-v$ data, free of line emission. The 68 channels were averaged, Fourier transformed to the image domain, and cleaned in a similar manner as the $\mathrm{H}$ I maps. Contours of the $1.4 \mathrm{GHz}$ map are shown superposed on the $\mathrm{H}$ I map in Figure 3.

Baars \& Wendker (1976) found as an upper limit for the continuum emission $S_{c}=3 \mathrm{mJy}$. We detect radio continuum emission from the nucleus of NGC 404 at the $20 \sigma$ level. The source is unresolved and has a flux density of $3.6 \pm 0.3 \mathrm{mJy}$. We find a position of $\alpha_{\mathrm{B} 1950.0}=01^{\mathrm{h}} 06^{\mathrm{m}} 39^{\mathrm{s}} .25 \pm 0.03$ and $\delta_{\mathrm{B} 1950.0}=35^{\circ} 27^{\prime} 07^{\prime \prime} .0 \pm 0 . \prime 3$. The errors are formal errors to the fit only. The radio and optical positions coincide to within the accuracy of the latter.

The radio power of the nuclear source (at an assumed distance of $3.3 \mathrm{Mpc})$ is given by $\log P_{21}\left(\mathrm{~W} \mathrm{~Hz}^{-1}\right)=17.9$. According to Hummel (1980) and Hummel \& Kotanyi (1982), about $80 \%$ of S0/a to Sab-type galaxies have a central continuum source with a total power similar to, or greater than, $\log P_{21}\left(\mathrm{~W} \mathrm{~Hz}^{-1}\right)=19.4$. In the study of S0 galaxies by van Driel \& van Woerden (1991, and references therein), $90 \%$ of the galaxies have a power higher than those indicated by Hummel (1980); even the lowest value found for their weakest object $\left(\log P=18.75\right.$ and assuming $\left.H_{0}=75 \mathrm{~km} \mathrm{~s}^{-1} \mathrm{Mpc}^{-1}\right)$ is more than 7 times higher than the value we find for NGC 404. The luminosity of the nuclear source is more than an order of magnitude lower than the Galactic supernova remnant Cas A and comparable to that of the Crab Nebula.

There are $\sim 50$ point sources with $S_{21}>3 \sigma$, but only one (besides the nuclear source) has $S_{21}>15 \sigma$. This source is located at the outer limit of the bright emission of $\mathrm{H}$ I and thus falls beyond the optical limit.

Maoz et al. (1998) found a nuclear UV spectrum characteristic of a very young $(<3 \mathrm{Myr})$, or at most moderately old ( $~ 5 \mathrm{Myr}$ ), burst of star formation. They conclude that the continuum UV source of this LINER is a stellar cluster. Its low luminosity could be explained with two to six O stars, although the real number probably is higher after correcting for extinction. With our results we can calculate the massive star formation rate (following Condon 1992) as $\operatorname{SFR}\left(M \geq 5 M_{\odot}\right.$ ) $\approx 2 \times 10^{-4} M_{\odot} \mathrm{yr}^{-1}$, a very low value if compared with the Milky Way $\left(0.3-0.5 M_{\odot} \mathrm{yr}^{-1}\right)$, E+A galaxies $\left(0.5 M_{\odot} \mathrm{yr}^{-1}\right.$, Miller \& Owen 2000), or even when compared with other S0's in which the massive star formation rate is of the order of $\sim 0.1 M_{\odot} \mathrm{yr}^{-1}$ (from data given by van Driel \& van Woerden 1991).

\section{ORIGIN OF THE H I}

The question about the origin of the gas in galaxies of Hubble type S0 has not been fully answered yet. For example, gas lost by evolving stars is likely to be retained by these fairly massive systems, offering a possible explanation for the origin of the gas. But in that case, one would expect a far larger proportion of $\mathrm{S} 0 \mathrm{~s}$ to be detected in $\mathrm{H}$ I. In the following we look at the different scenarios for explaining the origin (from internal as well as external sources) of the detected gas in NGC 404.

\subsection{Burnt-Out Disk}

This scenario assumes that lenticular galaxies are former spirals that consumed efficiently their gas content in the process of star formation (Larson et al. 1980). In that case, the gas-rich S0s could be lenticulars that, although they consumed the gas in the inner region, somehow retained some gas in the outskirts. This $\mathrm{H}_{\mathrm{I}}$ is then the leftover primordial gas from 
which the galaxy was formed. Because of the low density of the gas in the outer regions, stars never formed there.

Like many S0s, NGC 404 presents a central hole in its H I distribution giving it the appearance of a doughnut with an inner and outer radius of $\sim 1-4 R_{25}$. This extent is larger than that found in other S0s. The azimuthally averaged neutral atomic gas surface density, $\sigma_{\mathrm{HI}}$, in this ring is $\sigma_{\mathrm{HI}}=$ $1.2 M_{\odot} \mathrm{pc}^{-2}$, too low by at least a factor of 5 for a efficient star formation to proceed and of the same order as that encountered in many gas-rich S0's. We used Kennicutt's criterion (Kennicutt 1989) to look in a bit more detail into this issue. Using the observed one-dimensional velocity dispersion of the gas and observed rotation curve, we find that the gas surface density is too low by an order of magnitude for gravitational collapse of the gas to occur.

This implies that this scenario runs into trouble on various fronts. First, the neutral gas surface density is way too low for star formation to take place. In fact, one would expect not only that a close-to-critical surface density would be found but also that the peak gas density is reached at the edge of the stellar counterpart. In fact, we find it farther out, near $1.5 R_{25}$, i.e., far from the optical edge of the galaxy, which is where the purported SF zone ends. Also, within this framework other observed features of the gas, such as the change in position and inclination angles or the presence and morphology of the outer annulus, are difficult to account for. Hence, we consider it improbable that the H I gas in NGC 404 is mainly leftover primordial gas.

\subsection{Mass Loss of Evolved Stars}

It is well established that the ISM is constantly replenished through contributions of evolved stars, which inject gas and dust into the ISM. This process is also occurring in S0s and indeed, the amount of gas is not negligible: $d M / d t \sim 0.015$ $\left(L_{V} / 10^{9} L_{\odot}\right)\left(M_{\odot} \mathrm{yr}^{-1}\right)$ (Faber \& Gallagher 1976). This implies that the majority of S0s should be gas rich and that this is the rule rather than the exception. As we find the contrary, there must exist a mechanism that rids S0s from this gas. Star formation does not seem a viable candidate as little SF activity is recorded in S0s. Similarly, a galactic wind seems to be ruled out, as is interaction with, e.g., an intracluster medium, NGC 404 finding itself isolated. Alternatively, the gas that is lost inherits the velocity dispersion of the stars leading to temperatures in the $\mathrm{X}$-ray regime.

For a typical S0 with $M_{\mathrm{HI}} / L_{B} \lesssim 0.1 M_{\odot} / L_{\odot}$ and a blue luminosity $B$ of order $10^{9}$ to $10^{10} L_{\odot}$, we obtain a reasonable estimate for the time needed (a few times $10^{9} \mathrm{yr}$ ) to collect the observed amount of gas as a result of mass loss from evolved stars. However, in the case of NGC 404, its luminosity is very low $\left(\sim 7 \times 10^{8} L_{\odot}\right)$. This, combined with an $\mathrm{H}$ I mass-to-blueluminosity ratio higher than the majority of S0s studied thus far $\left(0.22 M_{\odot} / L_{\odot}\right)$, results in an estimated timescale of order a Hubble time. So although the ISM detected in NGC 404 could be accounted for theoretically by steady mass loss since it formed, there are serious shortcomings to this potential explanation. Mass loss by evolved stars would not answer the question why most of the ISM is found beyond $1.5 R_{25}$ either, where star formation is and has been negligible. Moreover, it would be difficult to explain where it got its angular momentum from.

\subsection{External Origin}

The former scenarios offer unsatisfactory explanations for the origin of $\mathrm{H}$ I in NGC 404. They especially fail to explain the fact that the outer ring is tilted. In fact, this is one of the stronger arguments for postulating an external origin of the gas.

The $\mathrm{H}$ I content could be the result of the merger of a dwarf irregular galaxy with NGC 404. If we assume $M_{\mathrm{HI}} / L_{B} \sim 0.6$ as a value representative for normal dwarf galaxies $(0.3 \leq$ $M_{\mathrm{HI}} / L_{B} \leq 1.0$; Huchtmeier \& Ehle 1999), we find that a dwarf with $M_{B} \sim-15.5$ has a large enough gas supply to account for the observed amount of $1.52 \times 10^{8} M_{\odot}$ of $\mathrm{H}$ I gas.

Assuming $M_{T} / L_{B} \sim 4 M_{\odot} / L_{\odot}$ (Huchtmeier \& Ehle 1999), the total mass of the dwarf in a single merger would have been $M_{T} \sim 10^{9} M_{\odot}$. The total mass of NGC 404 is at least $\sim 3 \times 10^{10} M_{\odot}$. The mass of the dwarf then would have been only $\sim 3 \%$ of that of NGC 404 , and the merger would have had no major dynamical effect on the latter.

In $\S 6$ we assumed that the gas could be described as moving in concentric annuli within the potential well of the galaxy. If left to their own design, such annuli tend to settle in a preferred plane, the plane of the galaxy. Following Tohline et al. (1982), we have calculated the timescale $\left(\tau_{d}\right)$ for settling to a preferred plane of two annuli in NGC 404, one at $R=200^{\prime \prime}$ and one at $R=800^{\prime \prime}$, assuming that the donor galaxy left its gas in a plane inclined by about $40^{\circ}$ with respect to NGC 404. For $R=800^{\prime \prime}, \tau_{d} \approx 3.5 \times 10^{9} \mathrm{yr}$, whereas for $R=200^{\prime \prime}, \tau_{d} \approx 0.9 \times 10^{9} \mathrm{yr}$ (which corresponds to $\sim 63$ rotational periods). As can be appreciated in Figures 6 (the plots of $i$ vs. radius) and 10 , the gas in the ring at $R=200^{\prime \prime}$ has already settled in the plane of the galaxy. Now if we assume that the gas has been accreted during just one interaction, this must have happened at least $0.9 \times 10^{9} \mathrm{yr}$ ago. This time lapse agrees fairly well with that necessary for the ring at $R=800^{\prime \prime}$ to settle from an original inclination of $i \sim 40^{\circ}$ to its current value of $i \sim 11^{\circ}$ (assuming Keplerian rotation). These arguments restrict considerably the time elapsed since the interaction. If it were much smaller than $0.9 \times 10^{9} \mathrm{yr}$, the gas of the inner ring (the doughnut) would not have had time to settle into the plane of the galaxy, whereas if it were much larger, the outer ring would have settled within the plane of the disk of NGC 404.

If we assume that the gas currently encountered in NGC 404 was transferred in a flyby encounter, the culprit must have moved outside of a volume with a radius of $1.1 \mathrm{Mpc}$ as there are no objects within 1.1 Mpc (Karachentsev et al. 2002). This would imply a relative velocity for the possible intruder of order $1200 \mathrm{~km} \mathrm{~s}^{-1}$, an uncomfortably high value, which rather argues in favor of a catastrophic merger.

In case the rotation curve is flat (model F), the dynamical timescale $\tau_{d}$ is slightly shorter, $\sim 0.5 \times 10^{9}$ yr for $R=200^{\prime \prime}$, and comes out to be $\sim 2 \times 10^{9}$ yr for $R=800^{\prime \prime}$.

\section{DISCUSSION}

Numerical simulations of hierarchical galaxy formation within the $\Lambda$ cold dark matter $(\Lambda C D M)$ paradigm and carried out with sufficient resolution (in mass) to distinguish dwarf galaxies (Klypin et al. 1999; Moore et al. 1999) predict the existence of numerous low-mass halos. Observations, however, come up with numbers much smaller than predicted. Among the possible explanations that have been invoked are that these halos are too small to produce star formation or that their luminosity is so low that this makes them difficult to detect. In those systems in which star formation has taken place, $M / L_{B}$ ratios tend to be high (Tully et al. 2002). These systems are thought to appear in groups. In contrast, NGC 404 is an isolated galaxy. Karachentsev et al. (2002) suggest that 
this galaxy may represent the final stage of consecutive merging of members of a former group of galaxies. In this case, it could be the dominant member of a group of small galaxies, some of which, rich in neutral gas, have contributed the material that extends up to almost $8 R_{25}$.

The high $M / L$ ratio confirms that this is an extreme case in which matter (bright and dark) is highly concentrated. This high mass concentration also fits in with the picture of the orbits of gas captured through a merger event decaying within a few gigayears and aligning with the plane of the galaxy (Casertano \& van Gorkom 1991). This implicitly assumes that there is no extended dark halo to maintain gas orbits in a warp for a long period of time.

Regarding the apparently falling rotation curve, there are several other galaxies (NGC 7793, Carignan \& Puche 1990; NGC 2683 and NGC 3521, Casertano \& van Gorkom 1991; a few galaxies among their 967 spiral galaxy sample, Persic \& Salucci 1995; NGC 4244, Olling 1996; NGC 4138, Jore et al. 1996; 11 galaxies, Honma \& Sofue 1997; the Milky Way, among others, Sofue et al. 1999; and NGC 7631 and KUG 2318+078, Chung et al. 2002) in which the rotation curve begins to decline close to $2 R_{25}$. However, in most of these cases the $\mathrm{H}$ I measurements do not go much further than this radius, and this might be why no convincingly declining rotation curves have been found to date.

So, Keplerian declines are rare. Honma \& Sofue (1997) list 11 galaxies for which with some confidence one can state that the rotation curve falls significantly (by $50-100 \mathrm{~km}$ $\mathrm{s}^{-1}$ ). The most extreme case, and in fact a system quite similar to NGC 404, is NGC 4138, which was observed by Jore et al. (1996). H I was detected out to 2.5 optical disk radii (16 scale lengths). This gas is counterrotating with respect to the main stellar body, suggesting recent capture of a gas-rich satellite, like NGC 404. But unfortunately, also in this case difficulties in fitting the rotation curve prevents the authors from unambiguously confirming a Keplerian decline.

In the case of NGC 404, nature is playing tricks on us and decided to put this object close to face-on, making it impossible to solve separately for the inclination and rotation speed in the plane of the galaxy. Hence, we decided to analyze two extreme options for the intrinsic rotation curve: a flat rotation curve out to the last measured point (model F) and a curve following a Keplerian decline (model $\mathrm{K}$ ).

If the rotation curve remains flat, then NGC 404 is one of the "darkest galaxies" known with an $M / L$ of 175 and a fraction of $92 \%$ of its mass being due to dark matter. It would also be one of very few galaxies for which a rotation curve out to $\sim 8 R_{25}$ (48 scale lengths in the $B$ band) has been determined. The only other galaxy for which data up to $\sim 8 R_{25}$ (15 scale lengths) are available is DDO 154 (Carignan \& Beaulieu 1989). On the other hand, if this galaxy really follows a Keplerian decline, it would be unique. In that case, the extent of the DM halo would reach out to $3.2 \mathrm{kpc}$ $\left(\sim 2 R_{25}\right)$. One argument that would favor Keplerian decline is that the warp geometry makes more sense and qualitatively fits the picture of accreted material settling within the gravitational potential of the galaxy, the outermost inclined annuli taking longer than the more inward located ones. An additional argument is that for model $\mathrm{K}$ the $M / L$ ratio is more reasonable.

Finally, the preference for a Keplerian decline is reinforced by the fact that in the case of a flat rotation curve, the inclination of the outer annulus is the same as that of the inner part of the galaxy. This is at odds with the shape of the outer annulus, which clearly gives the impression of having a larger ellipticity, which instead would imply a higher inclination. This is exactly what we find when fitting a Keplerian rotation curve. In this case we find that $68 \%$ of the mass in NGC 404 is in the form of dark matter.

\section{CONCLUSIONS}

NGC 404 is an exceptionally gas-rich early-type galaxy. The $\mathrm{H}_{\mathrm{I}}$ is distributed in two separate systems, a doughnut, which extends from $R_{25}$ or $100^{\prime \prime}$ to $4 R_{25}$ (1.6-6.4 kpc for a distance of $3.3 \mathrm{Mpc}$ ), and an annulus of much lower surface brightness, which extends from $4 R_{25}$ to roughly $8 R_{25}$.

We derive a total $\mathrm{H}$ I mass of $1.52 \pm 0.04 \times 10^{8} M_{\odot}$ of which $75 \%$ resides in the doughnut and the rest in the outer annulus. This translates to a distance-independent $M_{\mathrm{HI}} / L_{B}$ ratio of 0.22 in solar units, which is high for an S0 galaxy, since the median for S0's galaxies is $0.04 M_{\odot} / L_{\odot}$, whereas it is $\sim 0.22 M_{\odot} / L_{\odot}$ for $\mathrm{Sb}$ galaxies (Roberts \& Haynes 1994).

The velocity field shows a steeply declining rotation curve. Unfortunately, the galaxy is oriented almost face-on and, assuming circular rotation of the gas in annuli, good fits can be obtained when forcing both a flat rotation curve and a rotation curve that falls off like a Keplerian. In both cases the position angle (P.A.) of the kinematical major axis varies with radius by $\sim 40^{\circ}$. In the Keplerian decline model, the gas in the outer annulus is warped, the annulus moving to a less face-on orientation with radius.

A total mass of $3 \times 10^{10} M_{\odot}$ is found when using the Keplerian rotation curve. This implies a global $M_{T} / L_{B}$ ratio of $\sim 44$ in solar units, which is exceptionally high. This is partly because the blue luminosity of the galaxy is $\sim 15$ times lower than average for an S0. For a flat rotation curve, a lower mass limit of $12 \times 10^{10} M_{\odot}$ or 4 times higher is derived, leading to a consequently 4 times higher total mass-luminosity ratio of $\sim 175 M_{\odot} / L_{\odot}$.

The nuclear source is detected at $20 \mathrm{~cm}$. If nonthermal, its luminosity of $\log P_{21}\left(\mathrm{~W} \mathrm{~Hz}^{-1}\right)=17.9$ is a factor of 10 lower than Cas A and more in line with that of the Crab nebula. If dominated by star formation, the SFR is a low $\operatorname{SFR}\left(M \geq 5 M_{\odot}\right) \approx 2 \times 10^{-4} M_{\odot} \mathrm{yr}^{-1}$, two to six $6 \mathrm{O}$ stars being enough to explain the observed luminosity.

Given the mass and luminosity of NGC 404, we are dealing with a dwarf lenticular galaxy. Assuming Keplerian decline, the DM halo is compact and ends near $2 R_{25}$. Our preferred explanation for the origin of the ISM in this system is that it has been accreted through a catastrophic merger with a gas-rich, dwarf irregular system, less than a tenth of the mass of NGC 404, which took place anywhere between $0.5-1.0 \times 10^{9} \mathrm{yr}$ ago (no other objects having been encountered within a volume of $1.1 \mathrm{Mpc}$ radius). Gas at small radius has already settled within the plane, whereas gas in the outer annulus is still gravitating toward the midplane of the disk defined by the S0, resulting in a warp of the gas distribution.

Although none of our conclusions depends strongly on the rotation curve being flat or declining, it is of course extremely frustrating that it has not proven possible to decide between these two extreme models, especially since the observed radial velocities decline so strongly. This leaves NGC 404 as potentially the first galaxy with a rotation curve that shows such a pronounced decline over such a large extent, which if confirmed, would be of extreme importance. 
We gratefully acknowledge a careful reading by an anonymous referee, which has improved the presentation of this paper. This project was supported by Mexican CONACyT grants $33026-\mathrm{E}$ to M. S. d. R. and 27606-E to E. B. This research has made use of the NASA/IPAC Extragalactic Database, which is operated by the Jet Propulsion Laboratory, California Institute of Technology, under contract with the National Aeronautics and Space Administration.
Baars, J. W. M., \& Wendker, H. J. 1976, A\&A, 48, 405

Baggett, W. E., Baggett, S. M., \& Anderson, K. S. J. 1998, AJ, 116, 1626

Barbon, R., Capaccioli, M., \& Rampazzo, R. 1982, A\&A, 115, 388

Begeman, K. G. 1989, A\&A, 223, 47

Carignan, C., \& Beaulieu, S. 1989, ApJ, 347, 760

Carignan, C., \& Puche, D. 1990, AJ, 100, 394

Casertano, S., \& van Gorkom, J. H. 1991, AJ, 101, 1231

Chung, A., van Gorkom, J. H., O’Neil, K., \& Bothun, G. D. 2002, AJ, 123,2387

Condon, J. J. 1992, ARA\&A, 30, 575

de Vaucouleurs, G., de Vaucouleurs, A., Corwin, H. G., Jr., Buta, R. J., Paturel, G., \& Fouqué, P. 1991, Third Reference Catalogue of Bright Galaxies (Berlin: Springer)

Faber, S. M., \& Gallagher, J. 1976, ApJ, 204, 365

García, A. M. 1993, A\&AS, 100, 47

Honma, M., \& Sofue, Y. 1997, PASJ, 49, 539

Huchtmeier, W. K., \& Ehle, M. 1999, Publ. Astron. Soc. Australia, 16, 24

Humason, M. L., Mayall, N. U., \& Sandage, A. R. 1956, AJ, 61, 97

Hummel, E. 1980, A\&AS, 41, 151

Hummel, E., \& Kotanyi, C. G. 1982, A\&A, 106, 183

Jore, K. P., Broeils, A. H., \& Haynes, M. P. 1996, AJ, 112, 438

Jörsäter, S., \& van Moorsel, G. A. 1995, AJ, 110, 2037

Jura, M. 1986, ApJ, 306, 483

Karachentsev, I. D., et al. 2002, A\&A, 389, 812

Kennicutt, R. C., Jr. 1989, ApJ, 344, 685

Klypin, A., Kravtsov, A. V., Valenzuela, O., \& Prada, F. 1999, ApJ, 522, 82

Knapp, G. R., Guhathakurta, P., Kim, D. W., \& Jura, M. A. 1989, ApJS, 70,329

Larson, R. B., Tinsley, B. M., \& Caldwell, C. N. 1980, ApJ, 237, 692

Maoz, D., Koratkar, A., Shields, J. C., Ho, L. C., Filippenko, A. V., \& Stenberg, A. 1998, AJ, 116, 55

\section{REFERENCES}

Miller, N. A., \& Owen, F. N. 2000, in ASP Conf. Ser. 240, Gas and Galaxy Evolution, ed. J. E. Hibbard, M. Rupen, \& J. H. van Gorkom (San Francisco: ASP), 632

Moore, B., Ghigna, S., Governato, F., Lake, G., Quinn, T., Stadel, J., \& Tozzi, P. 1999, ApJ, 524, L19

Morgan, W. W. 1958, PASP, 70, 364

Olling, R. P. 1996, AJ, 112, 457

Persic, M., \& Salucci, P. 1995, ApJS, 99, 501

Roberts, M. S., \& Haynes, M. 1994, ARA\&A, 32, 115

Richter, O.-G., \& Huchtmeier, W. K. 1984, A\&A, 132, 253

Rogstad, D. H., Lockhart, I. A., \& Wright, M. C. H. 1974, ApJ, 193, 309

Sage, L. J. 1990, A\&A, 239, 125

Sandage, A. 1961, The Hubble Atlas of Galaxies (Washington: CIW)

Schmidt, A. A., Bica, E., \& Alloin, D. 1990, MNRAS, 243, 620

Shu, F. H., Stachnik, R. V., \& Yost, J. C. 1971, ApJ, 166, 465

Sofue, Y., Tutui, Y., Honma, M., Tomita, A., Takamiya, T., Koda, J., \& Takeda, Y. 1999, ApJ, 523, 136

Tohline, J. E., Simonson, G. F., \& Caldwell, N. 1982, ApJ, 252, 92

Tonry, J. L., Dressler, A., Blakeslee, J. P., Ajhar, E. A., Fletcher, A. B., Luppino, G. A., Metzger, M. R., \& Moore, C. B. 2001, ApJ, 546, 681

Tully, R. B., \& Fisher, J. R. 1977, A\&A, 54, 661

Tully, R. B., Somerville, R. S., Trentham, N., \& Verheijen, M. A. W. 2002, ApJ, 569, 573

van Driel, W., \& van Woerden, H. 1991, A\&A, 243, 71

van Gorkom, J. H., Knapp, G. R., Raimond, E., Faber, S. M., \& Gallagher, J. 1986, AJ, 91, 791

Walter, F., \& Brinks, E. 1999, AJ, 118, 273

Wiklind, T., \& Henkel, C. 1989, A\&A, 225, 1

-1990, A\&A, 227, 394 